\title{
Quiz: Pacemaker
}

Fragment of the ECG was recorded on the 12 leads Holter monitor ( Fig. 1).

Speed of the recording $-25 \mathrm{~mm} / \mathrm{s}$.
The permanent pacemaker in DDD mode was implanted to patient.

There are 5 morphologies of PQRST seen on this ECG.
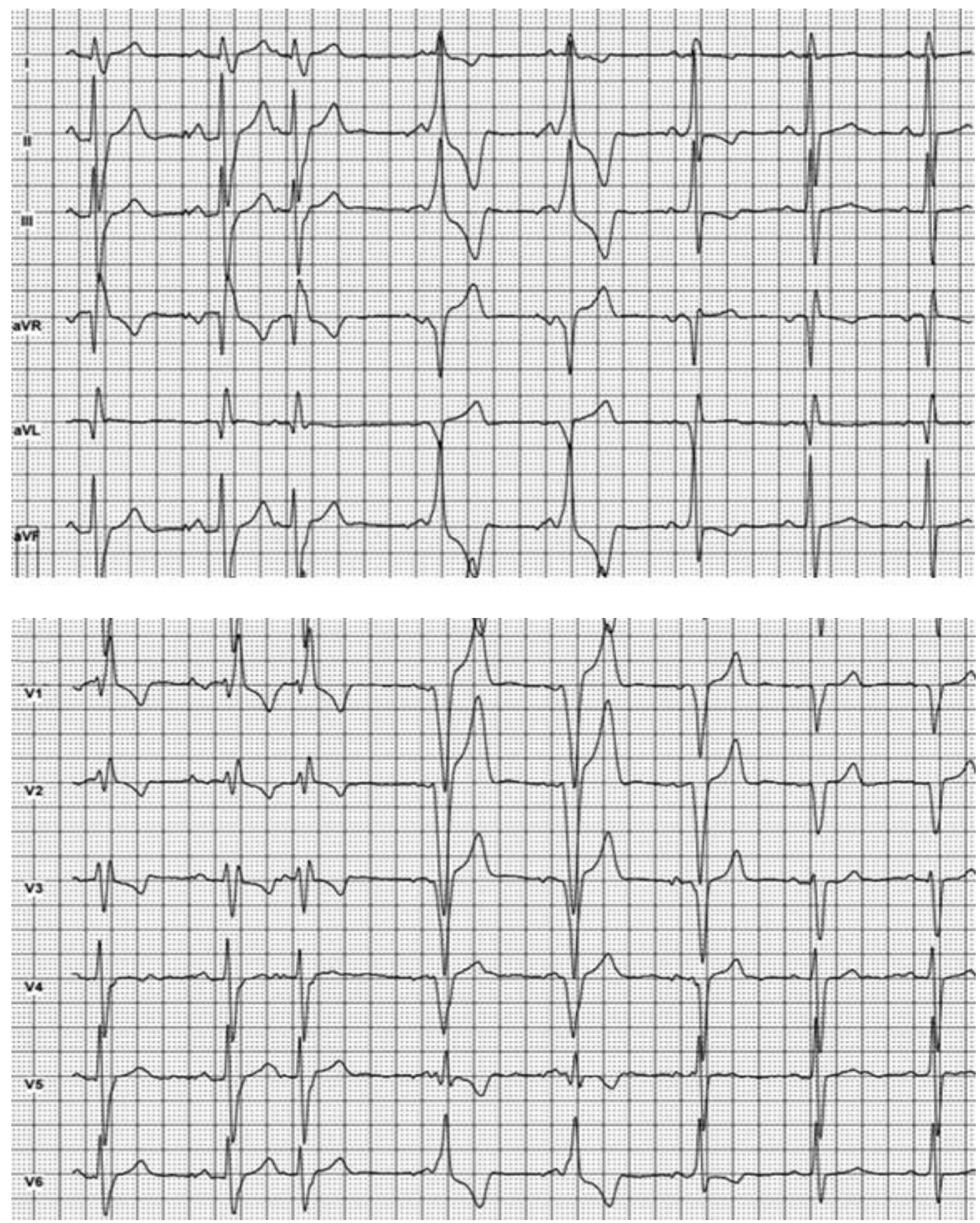

Figure 1. Multichannel Holter ECG recording ECG - electrocardiogram

Address for Correspondence: Mykhaylo Sorokivskyy, Lviv Regional Cardiology Center, Danylo Halytsky Lviv National Medical University, Lviv, Ukraine Email: msorokivskyy1@gmail.com

Received 25.10.2018 Accepted: 26.10.2018 doi: 10.24969/hvt.2018.87

Copyright $\bigcirc 2018$ Heart Vessels and Transplantation 
The correct description of the PQRST complexes (showed by order of numbers below) morphology on this ECG is:

A) 1,2 - atria: sinus rhythm, ventricles: paced rhythm; 3 - atria: premature beat, ventricles: paced rhythm; 4,5 - atria paced rhythm, ventricles: impulse conducted via $A V$ node with LBBB;

6 - atria and ventricles - fusion beat;

7,8 - atria and ventricles - sinus rhythm with incomplete LBBB.

B) 1,2 - atria: paced rhythm, ventricles: impulse conducted via AV node with RBBB;

3 - atria: premature beat, ventricles: impulse conducted via AV node with RBBB;

4,5 - atria: paced rhythm, ventricles: impulse conducted via AV node with LBBB;

6 - atria and ventricles - fusion beat;

7,8 - atria and ventricles - sinus rhythm with incomplete LBBB.

C) 1,2 - atria: paced rhythm, ventricles: impulse conducted via AV node with RBBB;

3 - atria: premature beat, ventricles: impulse conducted via AV node with RBBB;
4,5 - atria sinus rhythm, ventricles: impulse conducted through accessory pathway; 6 - atria and ventricles - fusion beat;

7,8 - atria and ventricles - sinus rhythm with incomplete LBBB.

D) 1,2 - atria: paced rhythm, ventricles: impulse conducted via AV node with RBBB;

3 - atria: premature beat, ventricles: impulse conducted via AV node with RBBB;

4,5 - atria: paced rhythm, ventricles: paced rhythm;

6 - atria and ventricles - fusion beat;

7,8 - atria and ventricles - sinus rhythm with incomplete LBBB.

Mykhaylo Sorokivskyy Lviv Regional Cardiology Center, Danylo Halytsky Lviv National Medical University, Lviv, Ukraine

Peer-review: Internal Conflict of Interest: None to Declare Authorship: M.S. Acknowledgement and funding: None to declare 